\title{
Photon equivalent charge in a two-electron temperature Fermi plasma
}

\author{
L. A. R IOS ${ }^{1}$, P. K. SHUKL A ${ }^{1}$ and A. SERBET O ${ }^{2}$ \\ ${ }^{1}$ Fakultät für Physik und Astronomie, Institut für Theoretische Physik IV, \\ Ruhr-Universität Bochum, D-44780 Bochum, Germany \\ (rios@if.uff.br) \\ ${ }^{2}$ Instituto de Física, Universidade Federal Fluminense, 24210-340 Niterói, \\ Rio de Janeiro, Brazil
}

(Received 3 March 2008 and in revised form 24 March 2008, first published online 24 October 2008)

\begin{abstract}
The equivalent photon charge in a two-electron temperature Fermi plasma is determined through the plasma physics method. The Fermi plasma has distinct populations of hot and cold electrons that are described by a quantum hydrodynamic model which accounts for the quantum statistical pressure of the hot electrons and the quantum force acting on the two electron fluids. Relations for the coupling between the electron plasma density fluctuations and the radiation fields are derived, and the effective photon charge is then calculated.
\end{abstract}

It is well known that an equivalent electric charge can be defined for an intense laser pulse propagating through a plasma $[1,2]$. In the case of electromagnetic radiation with a large spectral width, phase effects are negligible and the laser can be described as a gas of photons. Therefore, the photons can be considered as point-like particles, each moving with the group velocity of the laser and possessing an effective mass. The photon equivalent charge is a nonlinear concept associated with the ponderomotive force (radiation pressure) of the laser, which causes the polarization of the medium. It can lead to the photon Landau damping of the electron plasma waves [3]. The concepts of the effective mass and the equivalent charge of a photon can be described in the context of the quantum theory of radiation, and therefore are not just consequences of a classical description of the radiation-plasma coupling [4].

The induced photon charge is responsible for the coupling between electromagnetic waves and plasmas, which is important in space and astrophysical environments, as well as in solid-state physics. For instance, experiments making use of $\mathrm{X}$-rays are common in the dense matter community [5, 6], and allow accurate measurements of the physical properties of dense matter including temperature, density, and ionization state. Recently, Glenzer et al. [7] obtained the first collective $\mathrm{X}$-ray scattering measurements of plasmons in a warm dense plasma. In recent years there has been an increasing interest in investigating collective interactions in very dense Fermi plasmas, motivated by their applications in micro- and nano-scale objects, as well as in ultrasmall semiconductor devices [8]. Collective interactions in quantum plasmas can also be important in astrophysics [9]. In the interior of 
white dwarfs and in the crust of neutron stars the plasma is very dense and the electrons are degenerate. The electron Fermi temperature $T_{\mathrm{F}}$ is much higher than the plasma temperature $T$, which implies that quantum-mechanical effects are important $[10,11]$. A quantum hydrodynamic (QHD) model has been developed by Gardner and Ringhofer [12], which is able to describe a quantum electrostatic plasma in the collisionless regime, where collective, mean-field effects dominate [13]. Here the quantum-mechanical effects are described by the Bohm potential, which is related to the dispersion of the wave packet. For low-temperature Fermi plasmas, the electron distribution function is close to the Fermi-Dirac equilibrium and the Pauli blocking effect dramatically reduces the collision rates [10,11].

The existence of two distinct groups of electrons in laser-produced plasmas is well documented [14]. It has been proposed that energetic electrons produced by ultraintense lasers in plasmas could be used as an ignitor beam in a fast ignitor (FI) approach [15]. Conclusive evidence that space plasmas can also contain hot and cold electron components have been presented [16]. In two-electron plasmas, electronacoustic waves (EAWs) with wave frequencies larger than the ion plasma frequency can be generated $[17,18]$. These waves have phase velocities between the thermal speeds of the hot and cold electron populations, which means that the restoring force has its origin in the pressure of the hot electrons, while the inertia is provided by the mass of the cold population (ions form only a neutralizing background) [18].

In the present paper we determine the equivalent electric charge of photons in a super-dense two-electron temperature Fermi plasma. We derive the relations for the electron number density fluctuations driven by the ponderomotive force of the photons, and determine the induced photon charge. The above-mentioned relations are obtained by using the QHD model for the electrons, which accounts for the quantum statistical pressure law (hot electrons) and the quantum force associated with the quantum Bohm potential. The quantum potential represents part of the energy of the wave field and is related with quantum phenomena such as tunnelling from a potential well [19].

The dynamics of the electrostatic oscillations driven by the ponderomotive force of photons in a non-relativistic two-electron temperature Fermi plasma is governed by the continuity and momentum equations. The equations for the cold electrons are

and

$$
\frac{\partial n_{\mathrm{c}}}{\partial t}+\nabla \cdot\left(n_{\mathrm{c}} \mathbf{v}_{\mathrm{c}}\right)=0
$$

$$
\frac{\partial \mathbf{v}_{\mathrm{c}}}{\partial t}+\left(\mathbf{v}_{\mathrm{c}} \cdot \nabla\right) \mathbf{v}_{\mathrm{c}}=\frac{e \nabla \phi}{m_{\mathrm{e}}}+\frac{\hbar^{2}}{2 m_{\mathrm{e}}^{2}} \nabla\left[\frac{\nabla^{2}\left(\sqrt{n_{\mathrm{e}}}\right)}{\sqrt{n_{\mathrm{c}}}}\right]-\frac{e^{2} \nabla\left|\mathbf{E}_{0}\right|^{2}}{2 m_{\mathrm{e}}^{2} \omega_{0}^{2}},
$$

where $\phi$ is the electrostatic potential given by the Poisson equation

$$
\nabla^{2} \phi=4 \pi e\left(n_{\mathrm{e}}+n_{\mathrm{h}}-n_{0}\right),
$$

$n_{\mathrm{c}(\mathrm{h})}$ is the electron number density of the cold(hot) electron plasma and $n_{0}=n_{0 \mathrm{i}}$ is the equilibrium ion number density (ions are at rest). In the equilibrium, the quasi-neutrality condition $n_{0 \mathrm{c}}+n_{0 \mathrm{~h}}=n_{0 \mathrm{i}}=n_{0}$ is established, and $n_{0 \mathrm{c}(\mathrm{h})}$ is the equilibrium electron number density of the cold(hot) electrons. The second term in the right-hand side of (2) is the quantum force associated with the Bohm potential, where $\hbar$ is the Planck constant divided by $2 \pi, m_{\mathrm{e}}$ is the electron rest mass, and $c$ is the speed of light in vacuum. The last term in (2) is the ponderomotive force [20,21] 
due to the photon field, where $\mathbf{E}_{0}$ is the amplitude of the electric field associated with the electromagnetic wave packet, and $\omega_{0}$ is the frequency of the photons. For the hot electrons, we have

$$
\frac{\partial n_{\mathrm{h}}}{\partial t}+\nabla \cdot\left(n_{\mathrm{h}} \mathbf{v}_{\mathrm{h}}\right)=0
$$

and

$$
\frac{\partial \mathbf{v}_{\mathrm{h}}}{\partial t}+\left(\mathbf{v}_{\mathrm{h}} \cdot \nabla\right) \mathbf{v}_{\mathrm{h}}=\frac{e \nabla \phi}{m_{\mathrm{e}}}-\frac{v_{\mathrm{F}}^{2} \nabla n_{\mathrm{h}}^{3}}{5 n_{0 \mathrm{~h}}^{2} n_{\mathrm{h}}}+\frac{\hbar^{2}}{2 m_{\mathrm{e}}^{2}} \nabla\left[\frac{\nabla^{2}\left(\sqrt{n_{\mathrm{h}}}\right)}{\sqrt{n_{\mathrm{h}}}}\right]-\frac{e^{2} \nabla\left|\mathbf{E}_{0}\right|^{2}}{2 m_{\mathrm{e}}^{2} \omega_{0}^{2}} .
$$

The second term in the right-hand side of (5) is the force due to the pressure of a zero-temperature $\left(T \ll T_{\mathrm{F}}\right)$ Fermi-Dirac plasma [11], where $v_{\mathrm{F}}=\left(2 k_{\mathrm{B}} T_{\mathrm{F}} / m_{\mathrm{e}}\right)^{1 / 2}$ and $T_{\mathrm{F}}=\hbar^{2}\left(3 \pi^{2} n_{0 \mathrm{~h}}\right)^{2 / 3} / 2 k_{\mathrm{B}} m_{\mathrm{e}}$ are the Fermi speed and the Fermi temperature of the hot electrons, respectively, and $k_{\mathrm{B}}$ is the Boltzmann constant. The last two terms in the right-hand side of (5) are the quantum force and the ponderomotive force, respectively.

Since the EAWs have phase velocity smaller than the thermal speed of the hot electrons, we can neglect the electron inertia in (5). Therefore, linearizing (1)-(5) and combining the results, we have

$$
\left(\frac{\partial^{2}}{\partial t^{2}}+\frac{\hbar^{2} \nabla^{4}}{4 m_{\mathrm{e}}^{2}}\right) \delta n_{\mathrm{c}}+\omega_{\mathrm{pc}}^{2}\left(\delta n_{\mathrm{c}}+\delta n_{\mathrm{h}}\right)=\frac{e^{2} n_{0 \mathrm{e}} \nabla^{2}\left|\mathbf{E}_{\mathbf{0}}\right|^{2}}{2 m_{\mathrm{e}}^{2} \omega_{0}^{2}}
$$

and

$$
\left(\frac{\hbar^{2} \nabla^{4}}{4 m_{\mathrm{e}}^{2}}-C_{\mathrm{s}}^{2} \nabla^{2}\right) \delta n_{\mathrm{h}}+\omega_{\mathrm{ph}}^{2}\left(\delta n_{\mathrm{h}}+\delta n_{\mathrm{c}}\right)=\frac{e^{2} n_{0 \mathrm{~h}} \nabla^{2}\left|\mathbf{E}_{\mathbf{0}}\right|^{2}}{2 m_{\mathrm{e}}^{2} \omega_{0}^{2}},
$$

where we have introduced the definitions $\omega_{\mathrm{pe}}=\left(4 \pi e^{2} n_{0 \mathrm{c}} / m_{\mathrm{e}}\right)^{1 / 2}$ (cold electron plasma frequency), $\omega_{\mathrm{ph}}=\left(4 \pi e^{2} n_{0 \mathrm{~h}} / m_{\mathrm{e}}\right)^{1 / 2}$ (hot electron plasma frequency), $C_{\mathrm{s}}=$ $\left(6 k_{\mathrm{B}} T_{\mathrm{F}} / 5 m_{\mathrm{e}}\right)^{1 / 2} \equiv \sqrt{3 / 5} v_{\mathrm{F}}$ (electron thermal speed), and $\delta n_{\mathrm{c}}$ and $\delta n_{\mathrm{h}}$ are the cold electron and hot electron number density perturbations, respectively. Equations (6) and (7) show the coupling between the photon field and the density fluctuations in the two different electron components of the dense Fermi plasma.

In order to obtain the induced photon charge, we Fourier transform (6) and (7) and use the definition of the number density of photons as $n_{\mathrm{p}}=\left|\mathbf{E}_{0}\right|^{2} / 8 \pi \hbar \omega_{0}$. Manipulating the resultant equations, we have

$$
\begin{aligned}
\delta n_{\mathrm{e}} & =\delta n_{\mathrm{c}}+\delta n_{\mathrm{h}} \\
& =\frac{\hbar k^{2} \omega_{\mathrm{ph}}^{2}\left[\delta\left(k^{2} C_{\mathrm{s}}^{2}+k^{2} C_{\mathrm{Q}}^{2}\right)-\left(\omega^{2}-k^{2} C_{\mathrm{Q}}^{2}\right)\right] n_{\mathrm{p}}}{m_{\mathrm{e}} \omega_{0}\left[\left(\omega^{2}-k^{2} C_{\mathrm{Q}}^{2}\right)\left(k^{2} C_{\mathrm{s}}^{2}+k^{2} C_{\mathrm{Q}}^{2}+\omega_{\mathrm{ph}}^{2}\right)-\omega_{\mathrm{ph}}^{2} \delta\left(k^{2} C_{\mathrm{s}}^{2}+k^{2} C_{\mathrm{Q}}^{2}\right)\right]},
\end{aligned}
$$

where $\omega_{\mathrm{pc}}^{2}=\omega_{\mathrm{ph}}^{2} \delta, \delta=n_{0 \mathrm{c}} / n_{0 \mathrm{~h}}$ and $C_{\mathrm{Q}}=\hbar k / 2 m_{\mathrm{e}}$. Using (8) and the relation $-e \delta n_{\mathrm{e}}=q_{\mathrm{p}} n_{\mathrm{p}}[1]$, where $q_{\mathrm{p}}$ is the (spectral) equivalent photon charge, we derive

$$
q_{\mathrm{p}}=\frac{-e \hbar k^{2} \omega_{\mathrm{ph}}^{2}\left[\delta\left(k^{2} C_{\mathrm{s}}^{2}+k^{2} C_{\mathrm{Q}}^{2}\right)-\left(\omega^{2}-k^{2} C_{\mathrm{Q}}^{2}\right)\right]}{m_{\mathrm{e}} \omega_{0}\left[\left(\omega^{2}-k^{2} C_{\mathrm{Q}}^{2}\right)\left(k^{2} C_{\mathrm{s}}^{2}+k^{2} C_{\mathrm{Q}}^{2}+\omega_{\mathrm{ph}}^{2}\right)-\omega_{\mathrm{ph}}^{2} \delta\left(k^{2} C_{\mathrm{s}}^{2}+k^{2} C_{\mathrm{Q}}^{2}\right)\right]} .
$$

Figure 1 shows the dispersion relations of a Fermi plasma with $n_{0}=10^{28} \mathrm{~cm}^{-3}$ and of a two-electron temperature Fermi plasma, i.e.

$$
\omega=\left[\frac{\left(k^{2} C_{\mathrm{s}}^{2}+k^{2} C_{\mathrm{Q}}^{2}\right)\left(\delta+k^{2} \lambda_{\mathrm{Qh}}^{2}\right)+k^{2} C_{\mathrm{Q}}^{2}}{\left(k^{2} \lambda_{\mathrm{Dh}}^{2}+k^{2} \lambda_{\mathrm{Qh}}^{2}+1\right)}\right]^{1 / 2},
$$




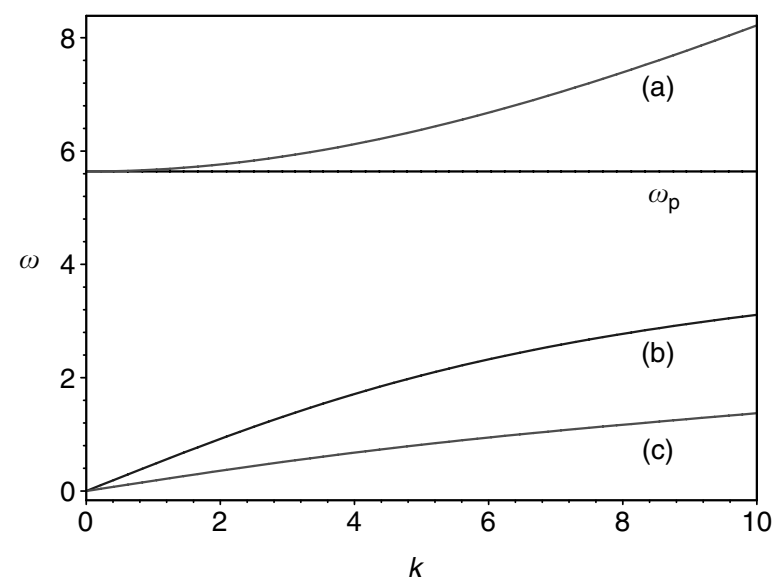

Figure 1. Frequencies (in units of $10^{18} \mathrm{~s}^{-1}$ ) for (a) a Fermi plasma with $n_{0}=10^{28} \mathrm{~cm}^{-3}$, and for a two-electron Fermi plasma with $n_{0}=10^{28} \mathrm{~cm}^{-3}$ and (b) $\delta=1$ and (c) $\delta=0.1$ ( $k$ is in units of $\left.10^{8} \mathrm{~cm}^{-1}\right)$.

for two different values of $\delta, 1$ and 0.1 , and $n_{0}=10^{28} \mathrm{~cm}^{-3}$. Here $\lambda_{\mathrm{Dh}}=C_{\mathrm{s}} / \omega_{\mathrm{ph}}$ is the Debye radius of the hot electrons and $\lambda_{Q h}=C_{Q} / \omega_{\text {ph }}$. From (10) we observe how the presence of the two distinct groups of electrons modifies the frequencies of the waves that can propagate in a dense Fermi plasma.

Defining $\omega_{\mathrm{p}}^{2}=\omega_{\mathrm{pc}}^{2}+\omega_{\mathrm{ph}}^{2}=\omega_{\mathrm{ph}}^{2}(1+\delta)$ and considering the limit $\omega^{2} \ll \omega_{\mathrm{p}}^{2}+k^{2} C_{\mathrm{s}}^{2}+$ $k^{2} C_{\mathrm{Q}}^{2}$, we find that

$$
q_{\mathrm{p}}=\frac{e \hbar k^{2}\left[\delta\left(\lambda_{\mathrm{Dh}}^{2}+\lambda_{\mathrm{Qh}}^{2}\right)+\lambda_{\mathrm{Qh}}^{2}\right]}{m_{\mathrm{e}} \omega_{0}\left[\left(\lambda_{\mathrm{Dh}}^{2}+\lambda_{\mathrm{Qh}}^{2}\right)\left(\delta+k^{2} \lambda_{\mathrm{Qh}}^{2}\right)\right]},
$$

where we have also considered $\left(k^{2} \lambda_{\mathrm{Dh}}^{2}+k^{2} \lambda_{\mathrm{Qh}}\right) \gg 1$.

To summarize, the equivalent photon charge in a two-electron temperature Fermi plasma has been determined by using the plasma physics method. We describe the electromagnetic wave packet propagating through the plasma as a gas of photons, and the QHD model is used to describe the two-electron temperature Fermi plasma. Here the quantum statistical pressure of the hot electron population and the quantum force associated with the Bohm potential are included. The radiation pressure of the photons causes the plasma polarization and the induced charge. A relation for the total electron number density fluctuation driven by the ponderomotive force of the photons is derived, and considering the spectral fluctuation in the total electron population we determine the equivalent photon charge in a two-electron temperature Fermi plasma. We observe that the quantum term $C_{\mathrm{Q}}$ can be important, depending on the value of $k$. The plasma composition also influences the dispersion relation of the waves in a dense Fermi plasma and, consequently, the spectral photon charge. These results can be important for the photon-plasma coupling in dense quantum plasmas, such as those in the interior of dense stars and in laser-solid density plasma interaction experiments.

\section{Acknowledgement}

One of the authors (L. A. Rios) was supported by a CNPq (Conselho Nacional de Desenvolvimento Científico e Tecnológico, Brazil) Postdoctoral Fellowship. 


\section{References}

[1] Mendonça, J. T., Silva, L. O., Bingham, R., Tsintsadze, N. L., Shukla, P. K. and Dawson, J. M. 1998 Phys. Lett. A 239, 373.

[2] Tsintsadze, N. L., Mendonça, J. T. and Shukla, P. K. 1998 Phys. Lett. A 249, 110.

[3] Bingham, R., Mendonça, J. T. and Dawson, J. M. 1997 Phys. Rev. Lett. 78, 247.

[4] Mendonça, J. T., Martins, A. M. and Guerreiro, A. 2000 Phys. Rev. E 62, 2989.

[5] Johnson, S. L., Heimann, P. A., Lindenberg, A. M., Jeschke, H. O., Garcia, M. E., Chang, Z., Lee, R. W., Rehr, J. J. and Falcone, R. W. 2003 Phys. Rev. Lett. 91, 157403.

Johnson, S. L., Heimann, P. A., Macphee, A. G., Lindenberg, A. M., Monteiro, O. R., Chang, Z., Lee, R. W. and Falcone, R. W. 2005 Phys. Rev. Lett. 94, 057407.

[6] Bergmann, U., Wernet, Ph., Glatzel, P., Cavalleri, M., Pettersson, L. G. M., Nilsson, A. and Cramer, S. P. 2002 Phys. Rev. B 66, 092107.

Glenzer, S. H., Gregori, G., Rogers, F. J., Froula, D. H., Pollaine, S. W., Wallace, R. S. and Landen, O. L. 2003 Phys. Plasmas 10, 2433.

[7] Glenzer, S. H. et al. 2007 Phys. Rev. Lett. 98, 065002.

[8] Kirichenko, O. V. and Peschansky, V. G. 2001 Low Temp. Phys. 27, 380.

Kornyushin, Y. and Que, W. 2002 J. Phys.: Condens. Matter 14, 10203.

Tilke, A. T., Simmel, F. C., Lorenz, H., Blick, R. H. and Kotthaus, J. P. 2003 Phys. Rev. B 68, 075311.

Plumridge, J. and Phillips, C. 2007 Phys. Rev. B 76, 075326.

[9] Adams, J. B., Ruderman, M. A. and Woo, C. 1963 Phys. Rev. 129, 1383.

Jung, Y.-D. 2003 Phys. Plasmas 10, 502.

Shukla, P. K. and Stenflo, L. 2006 Phys. Lett. A 355, 378.

Marklund, M. and Shukla, P. K. 2006 Rev. Mod. Phys. 78, 591.

Marklund, M. and Brodin, G. 2007 Phys. Rev. Lett. 98, 025001.

[10] Pines, D. 1961 J. Nucl. Energy C: Plasma Phys. 2, 5.

[11] Mandredi, G. 2005 Fields Inst. Commun. Ser. 46, 263.

[12] Gardner, C. L. and Ringhofer, C. 1996 Phys. Rev. E 53, 157.

[13] Manfredi, G. and Haas, F. 2001 Phys. Rev. B 64, 075316.

[14] Montgomery, D. S., Focia, R. J., Rose, H. A., Russell, D. A., Cobble, J. A., Fernández, J. C. and Johnson, R. P. 2001 Phys. Rev. Lett. 87, 155001.

Batani, D. et al. 2002 Phys. Rev. E 65, 066409.

Ferrante, G., Zarcone, M., Uryupina, D. S. and Uryupin, S. A. 2003 Phys. Plasmas 10, 3344.

[15] Tabak, M., Hammer, J., Glinsky, M. E., Kruer, W. L., Wilks, S. C., Woodworth, J., Campbell, E. M., Perry, M. D. and Mason, R. J. 1994 Phys. Plasmas 1, 1626.

[16] Lund, E. J., Labelle, J. and Treumann, R. A. 1994 J. Geophys. Res. 99 23,651.

Pottelette, R., Ergun, R. E., Treumann, R. A., Berthomier, M., Carlson, C. W., McFadden, J. P. and Roth, I. 1999 Geophys. Res. Lett. 26, 2629.

[17] Bezzerides, B., Forslund, D. W. and Lindman, E. L. 1978 Phys. Fluids 21, 2179.

Gary, S. P. and Tokar, R. L. 1985 Phys. Fluids 28, 2439.

[18] Yu, M. Y. and Shukla, P. K. 1983 J. Plasma Phys. 29, 409.

[19] Riggs, P. J. 2001 Erkenn. 68, 21.

[20] Yu, M. Y., Shukla, P. K. and Spatschek, K. H. 1974 Zh. Naturforsch. A 29, 1736.

Shukla, P. K., Yu, M. Y. and Spatschek, K. H. 1975 Phys. Fluids 18, 265.

[21] Shukla, P. K. and Stenflo, L. 1984 Phys. Rev. A 30, 2110.

Murtaza, G. and Shukla, P. K. 1984 J. Plasma Phys. 31, 423. 